\title{
Measuring the effects of video streaming quality in Virtual Reality experience
}

\author{
Sergo Martirosov ${ }^{1}$, Marko Matulin ${ }^{2}$ \\ ${ }_{1}$ University of West Bohemia, Mechanical faculty, Department of Industrial Engineering and \\ Management \\ Univerzitní 8, 306 14, Plzeň, Czech Republic \\ martiros@kpv.zcu.cz \\ ${ }_{2}$ University of Zagreb, Faculty of Transport and Traffic Sciences \\ Vukelićeva 4, 10000, Zagreb, Croatia \\ marko.matulin@fpz.hr
}

\begin{abstract}
Annotation: In this paper we present a methodology of measuring Virtual Reality (VR) experience while simulating various video streaming conditions. For that, we used four different videos, a VR headset and a video editing software, where specific changes were made to video clips in order to make it feel a believable video streaming experience. Frames per second(fps), resolution and bitrate variables were manipulated to measure the changes in perception of video quality. While streaming videos these variables get affected by the quality of internet connection. Our objective was to simulate such a scenario, where an internet connection quality would vary throughout the duration of the whole video, during which we could measure subjective score of quality perception.
\end{abstract}

\section{Introduction}

With the increase of internet speeds, it is becoming more and more popular to use Cloud technology to store and share data that can be easily accessed from anywhere in the world. Cloud Storage involves stashing data on hardware in a remote physical location, which can be accessed from any device via the internet. Cloud Computing is an on-demand computing model which relies on a stable Internet connection to share and access data across multiple devices such as computers or smartphones.

There is a good amount of research carried out on this topic. Advantages and challenges of Cloud Computing are described in more detail in [1]. Another research [2] suggests beneficial synergy between mobile platforms and Cloud Computing and tries to solve data synchronization issue while considering various network conditions. Furthermore, a study [3] discusses benefits for electronic and mobile learning as Cloud Computing service can have ubiquitous access through mobile devices and applications provided by cloud service providers.

In this controlled experiment we used videos stored locally. Those videos were altered to resemble realistic streaming scenario during experiment. Video is a visual multimedia source that combines a sequence of images to 
form a moving picture. There are many uses of videos such as entertainment, marketing, education, etc. Just by itself, video is proven to be a very powerful tool in teaching and learning. There are numerous studies conducted on this topic noting benefits of using videos in higher education and suggesting different ways to improve video-based teaching [4][5][6][7]. One study not only describes the benefits but also notes that different video lecture types can affect attention, emotion, cognitive load and learning performance [8].

In combination with Virtual Reality, the added immersion could increase video effectiveness even more for any of its purposes. In immersive Virtual Reality, the real world is completely occluded from the field of view and the user has a stronger feeling of presence in that virtual environment [9]. Examples of immersive Virtual Reality are Oculus Rift and HTC Vive.

In the modern time Virtual Reality (VR), technology became very popular and is used in many different areas such as entertainment, military, healthcare, education, engineering etc. VR is a simulated environment that is created with computer technology and presented to the user in such a way, that user starts to feel like in real environment. A Simulation is a model of a real world where user has the ability to interact with the environment [10]. Simulations are helpful and useful as they provide realistic context in which individuals can explore, experiment and see immediate results as they create models of their own or try theories on the modelled concept [11]. Depending on the amount of senses simulated in VR, such as vision, hearing, touch, balance, even smell, the immersion level in the artificial world can vary. Thanks to modern technological advance of computer hardware and software, it became possible to incorporate 3D Virtual Reality in innovative applications of teaching, training, and learning [12] [13] [14].

Interactivity is the most fundamental aspect in learning. It is mentioned that both, providing a learning environment and interaction with PCs may promote a better understanding of things [15]. Despite many studies in this area, there is still controversy about the real effects of VR on learning and cognition, but preliminary results indicate that the use of VR can increase learning performance [16], facilitate usability and enhance interaction [17], help to reconstruct and navigate through non-existent environments [18], help disabled learners with knowledge accessibility [19], etc. Serious games tend to be used often in formal education and with sufficient support are shown to be highly motivational and effective in learning complex tasks [20] [21].

\section{$2 \quad$ Video Streaming Parameters}

\subsection{BitRate}

A video bitrate is the number of bits that are processed in a unit of time. Video data rates are given in bits per second (bit/s). In general, a higher bitrate correlates with higher video quality, only when comparing the same video with the same resolution. Additionally, when the resolution goes up, bitrates should 
be expected to go up as well, as more data is being processed. Although high video bitrate may provide excellent quality, but it can also be very taxing on your hardware which can result in stutters.

A study suggests an encoding method of detecting a region of interest in a video to achieve trade off between bitrate quality and perceptual quality [22]. Region of interest is an area of the video a person will most likely look at. Another two studies try to find minimum bitrate requirements for sufficient user viewing experience [23] [24]. Lastly, researchers suggest that depending on network conditions, lower quality videos can actually yield better results in comparison to higher quality videos [25].

\subsection{Resolution}

Video resolution in a display device refers to the number of distinct pixels that could be displayed in each dimension. The resolutions you often see such as $360 p, 480 p, 720 p$, or $1080 p$ - represent the number of horizontal lines a video has from top to bottom. For comparison, a 720p HD video has 720 lines that are each 1,280 pixels wide, meaning that it is more than twice as sharp as the same video at $480 p$ and can be viewed on a much larger screen. As of March 2012, $1366 \times 768$ was the most common display resolution [26].

Some of the research in this area was focused on measuring the effects of different video resolution quality on visual performance [27], eyestrain [28] and quality of experience [29] [30].

\subsection{Frame Rate}

A frame rate is simply the frequency at which independent still images appear on the screen. The human eye can see as much as 10 or 12 frames per second (fps) and process them separately, while $16 \mathrm{fps}$ is already perceived as a motion [31]. In videos with lower frame rates movements appears jerky, while the characters move at unnatural speeds. Frame rate greatly impacts the style and viewing experience of a video.

It was found that frame rates have significant effect on human performance [32] [33] [34]. Furthermore, some research was done to examine the connection between perceptual video quality and different frame rates [35] [36]. A study was performed to determine preferred frame rates at a fixed bit rate for low bitrate video, suggesting various frame rates for different types of videos [37].

\section{$3 \quad$ Methodology}

For our experiment, we had to prepare four different videos. As a Virtual Reality device, we had a HTC Vive. The device uses two OLED panels, one per eye, each having a display resolution of $1080 \times 1200(2160 \times 1200$ combined pixels). Due to its $1080 \mathrm{p}$ max resolution, there was no point in using videos with higher resolution, as there would be no additional quality added to 
the video viewing experience. All $360^{\circ}$ videos were downloaded with an initial quality of $1080 p$ from YouTube, a video hosting service. To avoid any discomfort for participants, the duration of those videos didn't exceed more than 3 minutes. Furthermore, participants were viewing the videos in a seated position.

The first video was shown to participants just to get used to VR. It was downloaded with $1080 \mathrm{p}$ resolution and the settings were left unchanged. The content of the video was polar night and the northern lights in increased playback speed. Due to mostly static camera and only polar lights movement, the video didn't exhibit any discomfort for participants. Apart from the first video, three other videos were shown to each participant in different order every time, to eliminate any effects of one video type having on another.

The second video was used to prepare resolution variations. For this, 1080p, $720 p, 480 p$, and $360 p$ resolution variations of the same videos were downloaded. Then, in Adobe premiere pro, we had to cut clips from all types and finally stich them in one video. The content of the video was a guide for Buckingham Palace. The pace of the video was slow to moderate, with additional audio guide.

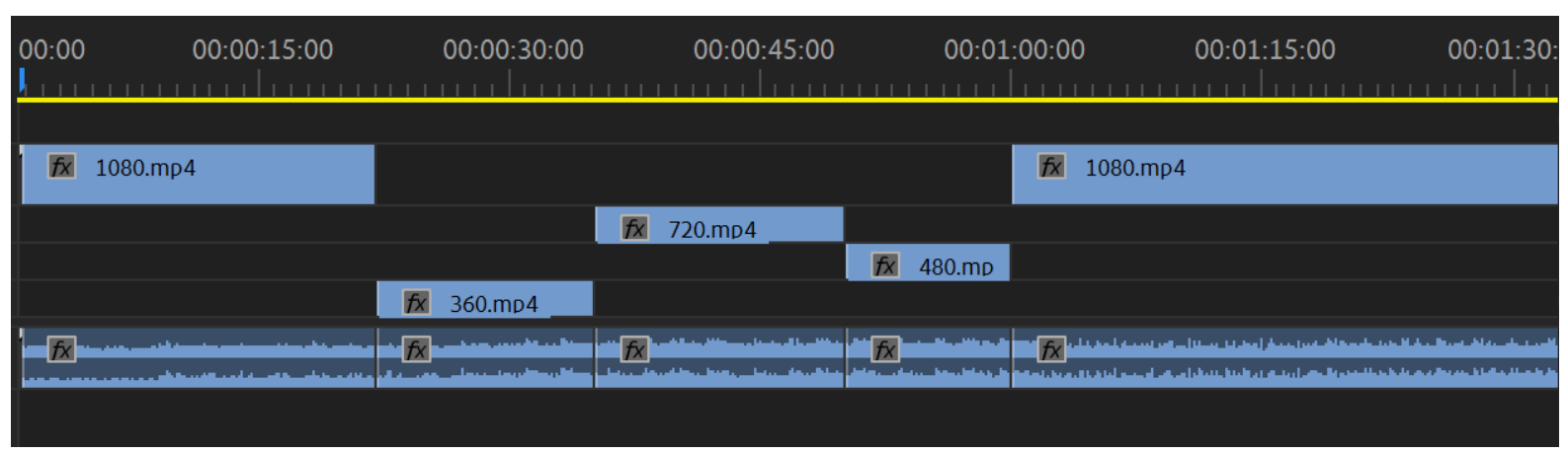

Figure 1 - Preparation of Resolution variation video in Adobe Premiere Pro

The third video was manipulated for frame drop simulation. Due to audio frame drop being so obvious to the ear and because we didn't want participants to easily notice changes in videos, audio lines were left untouched, while video itself was cut with different frame drops variations, which are, 1,2,3 and 4. These amounts were cut throughout the whole video in random timeline locations. The specified number of frames were first cut, then previous frames were copied on the missing frames location so that the picture doesn't become black while video player is on an empty frame timeline, but rather remain still until the next new untouched frame starts. On the image below, you can see a three-step procedure of cutting, removing and replacing empty frames with previous frames. The content of the video was Hong Kong tour with 1080p resolution, only frames were manipulated. 


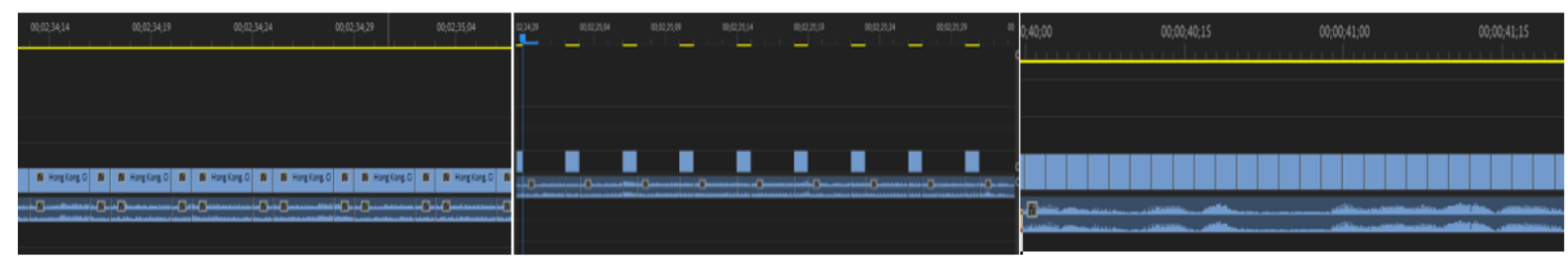

Figure 2 - Preparation of Frame drop video in 3 stages, cutting, deleting and replacing empty spots with previous frames

Lastly, the fourth video was used for bitrate variations. A 1080p resolution video was downloaded and in Adobe Premiere software, the video was exported with different bitrate settings. Those were 1,2, 3, 4, 5 and original 8 . Other variations (6 and 7 ) seemed to have almost no visual difference in comparison to the original, thus we decided to avoid unnecessary analysis. Same as resolution variation video, different pieces of bitrate videos were first cut and then stitched together in one video. Below you can see an image of the procedure in the software.

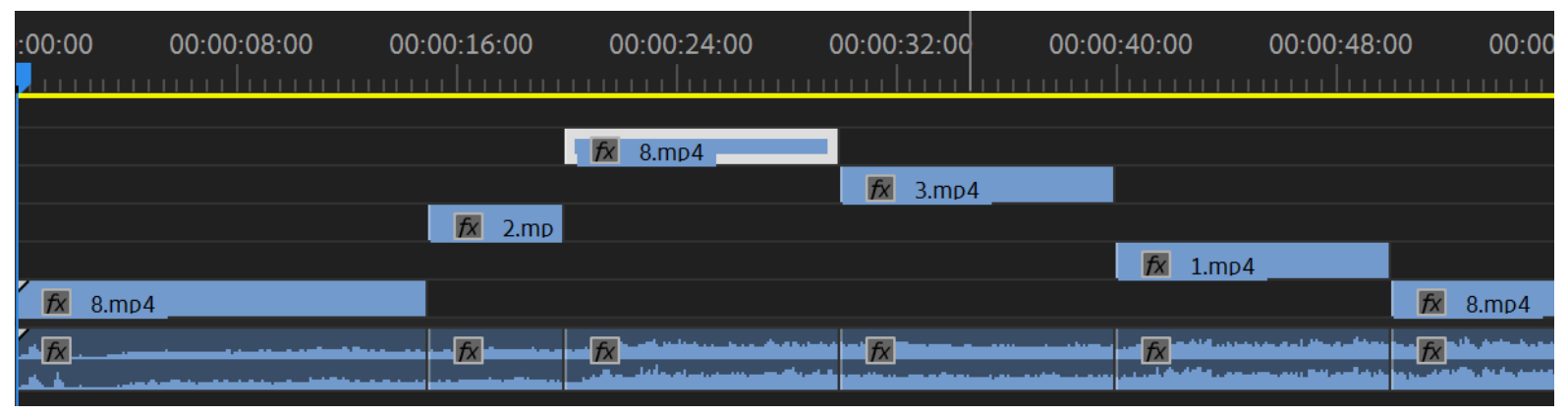

Figure 3 - Preparation of bitrate video

For this experiment we also prepared a subjective questionnaire that participants had to fill in after each video. During the videos, we were writing down all their subjective scores in a simple timeline table that we prepared in Microsoft Excel.

All in all, for the pilot study, we incorporated 20 students. If for the future analysis the gathered data will be insufficient, the experiment will continue and use more participants for larger database. Preliminary analysis suggests video resolution to have the most effect on quality of experience, then frame drop and lastly bitrate.

\section{Conclusion}

In this paper we presented a methodology that we used to measure quality of VR experience. During the experiment, participants had to watch several short videos, and throughout the duration of each video, give feedback on a scale of 1 to 5 , how they evaluate their experience. In the future works, a detailed analysis will be prepared of this study but as preliminary analysis suggests, there is a clear correlation between video quality and subjective feedback. Though, frame rate and resolution videos had more clear results than bit rate video. This could mean a not so big effect of bit rate on video quality for a 
person to notice, unless bitrate is too low. Furthermore, as we chose specific videos for this experiment, other types of videos could exhibit different results. In the future works we could find other types of videos and experiment with different variations of parameters.

\section{Acknowledgement}

This article was created with the subsidy of University of West Bohemia internal grant number SGS-2018-031 - "Optimizing sustainable production system parameters".

\section{References}

[1] J. Wu, L. Ping, X. Ge, Y. Wang and J. Fu, Cloud Storage as the Infrastructure of Cloud Computing, 2010 International Conference on Intelligent Computing and Cognitive Informatics, Kuala Lumpur, 2010, pp. 380-383. doi:10.1109/ICICCI.2010.119

[2] E. Lagerspetz and S. Tarkoma, Mobile search and the cloud: The benefits of offloading, 2011 IEEE International Conference on Pervasive Computing and Communications Workshops (PERCOM Workshops), Seattle, WA, 2011, pp. 117-122. doi:10.1109/PERCOMW.2011.5766852

[3] A. Ghazizadeh, Cloud Computing Benefits and Architecture in E-Learning, 2012 IEEE Seventh International Conference on Wireless, Mobile and Ubiquitous Technology in Education, Takamatsu, 2012, pp. 199-201. doi: 10.1109/WMUTE.2012.46

[4] Cynthia J. Brame, Effective Educational Videos: Principles and Guidelines for Maximizing Student Learning from Video Content, CBE-Life Sciences Education Vol. 15, No. 4, 2017, doi:10.1187/cbe.16-03-0125

[5] I. Vieira, A.P. Lopes, F. Soares (2014), The potential benefits of using videos in higher education, Edulearn14, Proceedings, pp. 750-756. ISBN: 978-84617-0557-3, ISSN: 2340-1117

[6] Yousef, Ahmed Mohamed Fahmy \& Amine Chatti, Mohamed \& Schroeder, Ulrik. (2014). Video-Based Learning: A Critical Analysis of The Research Published in 2003-2013 and Future Visions. eLmL - International Conference on Mobile, Hybrid, and On-line Learning.

[7] Woolfitt Z., The effective use of video in higher education, Lectoraat Teaching, Learning and Technology Inholland University of Applied Sciences, 2015.

[8] W. Wang, C. Chen and C. Wu, Effects of Different Video Lecture Types on Sustained Attention, Emotion, Cognitive Load, and Learning Performance, 2015 IIAI 4th International Congress on Advanced Applied Informatics, Okayama, 2015, pp. 385-390. doi:10.1109/IIAl-AAI.2015.225

[9] Merel T. (2015), the 7 drivers of the $\$ 150$ billion AR.VR industry. Aol tech; Available from https://techcrunch.com/2015/07/08/the-7-drivers-of-the-150billion-arvr-industry 
[10] Thurman, R.A. Instructional simulation from a cognitive psychology viewpoint, Educational Technology Research and Development December 1993, Volume 41, Issue 4, pp 75-89, doi:10.1007/BF02297513

[11] Rieber, L.P. Computer-based microworlds: A bridge between constructivism and direct instruction. Educational Technology Research and Development March 1992, Volume 40, Issue 1, pp 93-106, doi:10.1007/BF02296709

[12] A. G. Abulrub, A. N. Attridge and M. A. Williams, Virtual reality in engineering education: The future of creative learning, 2011 IEEE Global Engineering Education Conference (EDUCON), Amman, 2011, pp. 751-757. doi:10.1109/EDUCON.2011.5773223

[13] Gregory, S., Gregory, B., Reiners, T., Fardinpour, A., Hillier, M., Lee, M. J. W., ... Larson, I. (2013). Virtual worlds in Australian and New Zealand higher education: remembering the past, understanding the present and imagining the future. In H. Carter, M. Gosper, \& J. Hedberg (Eds.), Electric Dreams: 30th Ascilite Conference 2013 Proceedings (pp. 312-324). North Ryde, NSW: Macquarie University. ISBN 9781741384031

[14] Jou, Min \& Wang, Jingying. (2013). Investigation of effects of virtual reality environments on learning performance of technical skills. Computers in Human Behavior. 29. 433-438. doi:10.1016/j.chb.2012.04.020.

[15] Seymour, P., Nicholas, N., The Connected Family: Bridging the Digital Generation Gap, Longstreet Press, (1996). ISBN - 1563523353

[16] Carpenter T. \& Anderson L. (1996). Using virtual reality technology for learning design skills, Proceedings of ED-MEDIA 96, Boston, USA, pp.752

[17] Mikropoulos T.\& Nikolou E. (1996). A virtual hand with tactile feedback for virtual learning environments, Proceedings of ED-MEDIA 96, Boston, USA, pp. 792

[18] Littman M. (1996). Enhancing instruction through virtual reality, Proceedings of ICTE, New Orleans, USA,pp. 31-33

[19] Lumbreras M., Sánchez J., Barcia M. (1996), A 3D sound hypermedial system for the blind. In Proceedings of the First European Conference on Disability, Virtual Reality and Associated Technologies, pp.187-191, Maidenhead, UK.

[20] Amine Chatti, Mohamed \& Dyckhoff, Anna \& Schroeder, Ulrik \& Thüs, Hendrik. (2012). "A Reference Model for Learning Analytics". International Journal of Technology Enhanced Learning. 4. 318-331. doi:10.1504/IJTEL.2012.051815.

[21] Eseryel, D., Law, V., Ifenthaler, D., Ge, X., \& Miller, R. (2014). An investigation of the interrelationships between motivation, engagement, and complex problem solving in game-based learning. Journal of Educational Technology \& Society, 17(1), pp.42-53. ISSN: 11763647.

[22] Azad, Salahuddin \& Song, Wei \& Tjondronegoro, Dian. (2011). Measuring Bitrate and Quality Trade-Off in a Fast Region-of-Interest Based Video Coding. 442-453. 10.1007/978-3-642-17829-0_42.

[23] Song, Wei \& Tjondronegoro, Dian \& Docherty, Michael. (2011). Saving Bitrate vs. Pleasing Users: Where is the break-even point in mobile video 
quality? MM'11 - Proceedings of the 2011 ACM Multimedia Conference and Co-Located Workshops. 403-412. doi:10.1145/2072298.2072351.

[24] Winkler, Stefan \& Faller, Christof. (2006). Perceived Audiovisual Quality of Low-Bitrate Multimedia Content. IEEE Transactions on Multimedia. 8. doi:10.1109/TMM.2006.879871.

[25] D. C. Mocanu, A. Liotta, A. Ricci, M. T. Vega and G. Exarchakos, When does lower bitrate give higher quality in modern video services? 2014 IEEE Network Operations and Management Symposium (NOMS), Krakow, 2014, pp. 1-5. doi:10.1109/NOMS.2014.6838400

[26] Higher screen resolutions more popular for exploring the internet according to OneStat.com. [Retrieved 2019-08-12] techpowerup.com.

[27] Ziefle, Martina. (1999). Effects of Display Resolution on Visual Performance. Human factors. 40. 554-68. doi:10.1518/001872098779649355.

[28] M. S. Bhatti, W. Rasheed, T. B. Tang and A. Laude, Investigating eye-strain due to prolonged exposure to low resolution multimedia using LSFG, 2016 6th International Conference on Intelligent and Advanced Systems (ICIAS), Kuala Lumpur, 2016, pp. 1-4. doi:10.1109/ICIAS.2016.7824096

[29] T. Zinner, O. Hohlfeld, O. Abboud and T. Hossfeld, Impact of frame rate and resolution on objective QoE metrics, 2010 Second International Workshop on Quality of Multimedia Experience (QoMEX), Trondheim, 2010, pp. 29-34. doi:10.1109/QOMEX.2010.5518277

[30] A. Asan, W. Robitza, I. Mkwawa, L. Sun, E. Ifeachor and A. Raake, Impact of video resolution changes on QoE for adaptive video streaming, 2017 IEEE International Conference on Multimedia and Expo (ICME), Hong Kong, 2017, pp. 499-504. doi:10.1109/ICME.2017.8019297

[31] Read, Paul; Meyer, Mark-Paul; Gamma Group (2000). Restoration of motion picture film. Conservation and Museology. Butterworth-Heinemann. pp. 2426. ISBN 978-0-7506-2793-1

[32] F. Janzen, Benjamin \& Teather, Robert. (2014). Is 60 FPS better than 30 ? The impact of frame rate and latency on moving target selection. Conference on Human Factors in Computing Systems - Proceedings. doi:10.1145/2559206.2581214.

[33] Thropp, Jennifer E. and Jessie Y. C. Chen. The Effects of Slow Frame Rates on Human Performance. (2006).

[34] T. Claypool, Kajal \& Claypool, Mark. (2007). On frame rate and player performance in first person shooter games. Multimedia Syst. 13. pp. 3-17. doi:10.1007/s00530-007-0081-1.

[35] Liu, Yutao \& Zhai, Guangtao \& Zhao, Debin \& Liu, Xianming. (2015). Frame Rate and Perceptual Quality for HD Video. pp. 497-505. doi:10.1007/978-3319-24078-7_50.

[36] Banitalebi Dehkordi, Amin \& Pourazad, Mahsa \& Nasiopoulos, Panos. (2015). The Effect of Frame Rate on 3D Video Quality and Bitrate. 3D Research. 6. doi:10.1007/s13319-014-0034-3. 
[37] G. Yadavalli, M. Masry and S. S. Hemami, Frame rate preferences in low bit rate video, Proceedings 2003 International Conference on Image Processing (Cat. No.03CH37429), Barcelona, Spain, 2003, pp. I-441. doi:

10.1109/ICIP.2003.1246993 